\title{
Influences of Venus' topography on fully developed superrotation and near-surface flow
}

\author{
Masaru Yamamoto ${ }^{1}$ and Masaaki Takahashi ${ }^{2}$ \\ ${ }^{1}$ Research Institute for Applied Mechanics, Kyushu University, 6-1 Kasuga-Kouen, Kasuga 816-8580, Japan \\ ${ }^{2}$ Center for Climate System Research, University of Tokyo, 5-1-5 Kashiwanoha, Kashiwa 277-8568, Japan
}

(Received August 11, 2009; Revised October 20, 2009; Accepted October 24, 2009; Online published November 16, 2009)

\begin{abstract}
We investigate the influence of topography on Venus' atmospheric general circulation. Based on comparative simulations with and without the Venusian topography, we elucidate the role of the topography in the fully developed superrotation. Orographically forced stationary waves are predominant over Mt. Maxwell: slightly weakening the superrotation near the cloud top. Differently from previous GCM results, the orographically forced waves do not produce significant asymmetry between the northern and southern hemispheric superrotations in the present model. Weak surface flows from mountains to lowlands are caused by the pressure dependence of the Newtonian cooling. The pattern and magnitude of the near-surface flow are largely different from those simulated in the Herrnstein and Dowling (2007) model. This implies that the parameterizations of physical processes (such as Newtonian cooling, turbulence, diffusion, and surface drag) and the model resolution could significantly influence the pattern and magnitude of the near-surface flow and the orographical forcing of planetary-scale stationary waves.
\end{abstract}

Key words: Venus, topography, general circulation, superrotation, orographically-forced wave.

\section{Introduction}

Venus' atmospheric environment, consisting of dense $\mathrm{CO}_{2}$, is largely different from that on the Earth, a planet which has almost the same size. The surface atmospheric temperature and pressure have high values of $730 \mathrm{~K}$ and 92 bar, respectively. Optically thick clouds of sulfuric acid globally cover the planet in a height range of 50-70 km. Short-wave radiative heating by the cloud layer is one of driving forces of the atmospheric circulation of Venus. The solid part of Venus slowly rotates with a period of 243 days (Earth days) in an inertial frame, while the atmosphere near the cloud top $(65-70 \mathrm{~km})$ rapidly rotates with periods of $\sim 4$ days. Atmospheric rotation with an angular velocity faster than the planetary rotation is termed "superrotation". The cloud-top wind speed is approximately $100 \mathrm{~m} \mathrm{~s}^{-1}$, which is 60 times faster than the planetary rotation.

Although Atmospheric General Circulation Models (AGCMs) of the Venus atmosphere have been developed, it is difficult to incorporate the physical processes in AGCMs because of many uncertainties. Recently, Venuslike AGCMs simplifying the physical processes used for investigating Venus superrotation (Yamamoto and Takahashi, 2003; Lee et al., 2005; Hollingsworth et al., 2007; Herrnstein and Dowling, 2007). The formation and maintenance mechanisms of the superrotation are proposed on the basis of analyzing the simulated atmospheric circulation and waves. Their simulated superrotations are maintained by the Gierasch mechanism (Gierasch, 1975), in

Copy right(c) The Society of Geomagnetism and Earth, Planetary and Space Sciences (SGEPSS); The Seismological Society of Japan; The Volcanological Society of Japan; The Geodetic Society of Japan; The Japanese Society for Planetary Sciences; TERRAPUB which the meridional circulation pumps up the angular momentum required to maintain the superrotation with the help of waves with equatorward momentum fluxes (e.g., Rossow and Williams, 1979). According to Yamamoto and Takahashi $(2004,2006 a)$, thermal tides are forced by solar heating with a period of a Venusian day (117 Earth days) near the cloud top, contributing to the Gierasch mechanism through the equatorial acceleration of mean zonal flow (e.g., Newman and Leovy, 1992). Kelvin and Rossby waves are generated by horizontal shear instability (e.g., Iga and Matsuda, 2005) in the lower atmosphere, also contributing to the Gierasch mechanism. Although only a simulation with topography was reported in Yamamoto and Takahashi (2006b), the comparative simulations with and without topography were not conducted under the same physical condition in our General Circulation Model (GCM). We have conducted numerical experiments with and without topography in order to evaluate topographical effects on the GCM simulation. We have elucidated the influences of the topography on fully developed superrotation and near-surface flow by comparing the results of our two experiments and by also comparing these with recent other GCM studies (Lee, 2006; Herrnstein and Dowling, 2007).

\section{Model}

The model used is based on the version 5.6 of the AGCM developed at the Center for Climate System Research/National Institute for Environmental Study (Numaguti et al., 1997) and is similar to the model of Yamamoto and Takahashi $(2004,2006 a)$ (Exp. YT04). The dynamical process is calculated in the spectral space, while the physical process is calculated in the grid space of 


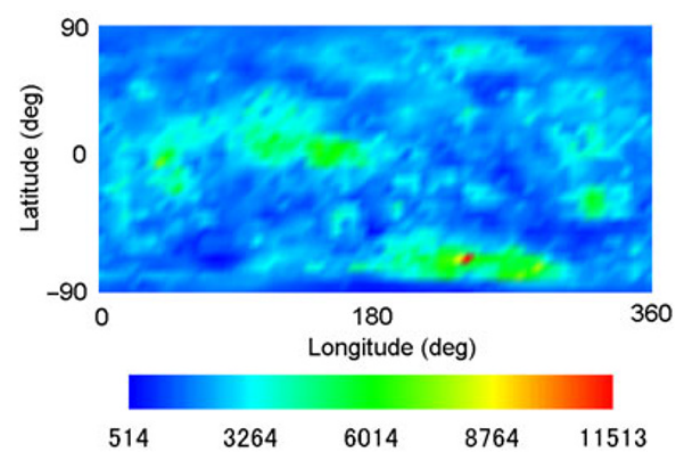

Fig. 1. Longitude-latitude distributions of ground surface elevation (m) obtained from Magellan topography data. The surface moves from left to right in the panel.

Table 1. Model parameters. $r$ indicates the planetary radius.

\begin{tabular}{lccc}
\hline Exp. & T & NT & YT04 \\
\hline Topography & Yes & No & No \\
$r(\mathrm{~km})$ & 6049.358 & 6049.358 & 6050 \\
$\tau_{\mathrm{H}}(\mathrm{h})$ & 60 & 60 & 96 \\
Surface drag & $C_{\mathrm{D}}\left(4 \times 10^{3}\right)$ & $C_{\mathrm{D}}\left(4 \times 10^{3}\right)$ & $\tau_{\text {drag }}(3$ days $)$ \\
\hline
\end{tabular}

$64 \times 32$. The truncation wavenumber of spherical harmonic function is 21 , and the vertical domain between 0 and $90 \mathrm{~km}$ has 52 sigma levels. The radiative processes are simplified by the 3D solar heating and the Newtonian cooling for long-wave radiation, of which the rates are the same as those in Exp. YT04. The maximum solar heating rate given in this model is $30 \mathrm{~K} \mathrm{day}^{-1}$ at the subsolar point and $65-\mathrm{km}$ altitude. The zenith-angle $(\lambda)$ dependence of the solar heating is $\cos ^{1.4} \lambda$. The equator-pole contrast of the surface potential temperature is set at $10 \mathrm{~K}$. Although both the heating rate below $55 \mathrm{~km}$ and surface temperature contrast are unrealistically large (figure 1 in Yamamoto and Takahashi, 2006a), the large rate and contrast are needed to reproduce fully developed superrotation. In the present work, the Magellan topography data (Ford and Pettengill, 1992) shown in Fig. 1 are given at the bottom boundary for Exp. T, but not for Exp. NT. The differences in model setup with respect to Exp. YT04 are listed in Table 1, and summarized as follows.

In Exp. T and Exp. NT, we set the drag coefficient $C_{\mathrm{D}}$ of $4 \times 10^{3}$ for temperature and horizontal flow (Del Genio et al., 1993) in order to introduce the effect of the wind speed on the surface drag. The 4 th order horizontal diffusion of the $e$-folding time $\tau_{\mathrm{H}}$ of $60 \mathrm{~h}$ at the maximum wavenumber is set in order to reduce the grid-scale eddies, which might be forced by topography and inertial instability. The diffusion is 1.6-fold larger than that in Exp. YT04. The initial surface pressure is obtained from the elevation by assuming the hydrostatic equation. In addition, the vertical profiles of the solar heating, Newtonian cooling, and reference atmospheric temperature (Fig. 2) are given as a function of pressure in Exp. T and Exp. NT, while they are given as a function of sigma in Exp. YT04. Although the surface temperature changes with changes in topography elevation in Exp. T, the surface potential temperature is the same in Exp. NT and Exp. TY04.

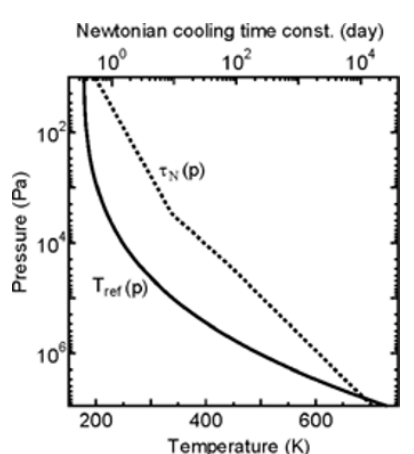

Fig. 2. Vertical distributions of a reference temperature $T_{\text {ref }}(p)$ (solid curve) and time constant of Newtonian cooling $\tau_{\mathrm{N}}(p)$ (dashed curve), which are functions of atmospheric pressure $p$.

\section{Results}

The zonal mean and eddy fields were investigated using the data sampled over $3072 \mathrm{~h}$ with a 3-h interval on Day 101,205. Figures 3(a) and (b) show longitude-latitude distributions of eddy temperature and horizontal flow averaged over a Venusian day at the undermost layer in Exp. T and Exp. NT. The eddy temperature and zonal-flow components of $\omega=0$ for a sampling period of 117 days (where $\omega$ is frequency) correspond to that of stationary waves. The eddy temperature decreases with elevation in Exp. T, but not in Exp. NT. The Newtonian cooling (which is a function of pressure) is larger with elevation, and thus air parcels near the surface become cooler at the top of mountain, compared with lowland areas. In this situation, the horizontal winds with speeds of 1-3 $\mathrm{m} \mathrm{s}^{-1}$ flow from the mountains to the lowlands. On the other hand, the stationary eddy components of horizontal wind and temperature have small values of $<1 \mathrm{~m} \mathrm{~s}^{-1}$ and $<0.15 \mathrm{~K}$, respectively, in Exp. NT. The pressure dependence of the Newtonian cooling induces the surface winds flowing from the mountains to the lowlands.

Figure 4(a) shows longitude-height distributions of eddy zonal flows averaged over a Venusian day (117 days) at $2.7^{\circ}$ latitude over the Aphrodite Terra around $120^{\circ}$ longitude. We can find stationary waves with high zonalwavenumber and amplitude of $10 \mathrm{~m} \mathrm{~s}^{-1}$ in Exp. T. The stationary waves propagate vertically in the upper regions above $65 \mathrm{~km}$ (where the atmosphere is highly stable) and have standing phase structures and an amplitude of about $3 \mathrm{~m} \mathrm{~s}^{-1}$ in the lower atmosphere below $65 \mathrm{~km}$ (where the atmospheric stability is low). Since the lower atmospheric waves have amplitudes of $5 \mathrm{~m} \mathrm{~s}^{-1}$ over the high mountain areas (around $30^{\circ}$ and $120^{\circ}$ longitudes), they are orographically forced. On the other hand, stationary waves are not generated in the equatorial region for Exp. NT.

Figure 4(b) shows longitude-height distributions of eddy zonal flows averaged over a Venusian day (117 days) at $-69.2^{\circ}$ latitude. Planetary-scale stationary waves are orographically forced over Mt. Maxwell around $240^{\circ}$ longitude, as shown in Exp. T. The orographically forced waves have standing phase structures below $50 \mathrm{~km}$ and propagate vertically above $50 \mathrm{~km}$. On the other hand, planetary-scale waves with an amplitude of $>10 \mathrm{~m} \mathrm{~s}^{-1}$ are found above $40 \mathrm{~km}$ in Exp. NT. They are not orographically forced, but are probably generated by horizontal shear instability. 


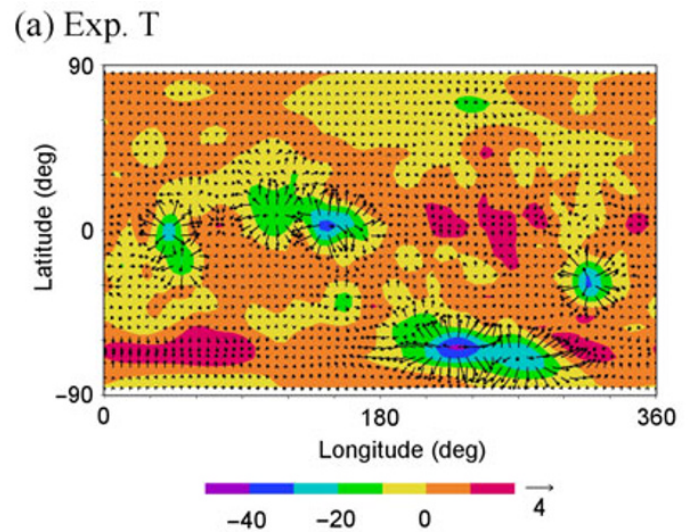

(b) Exp. NT

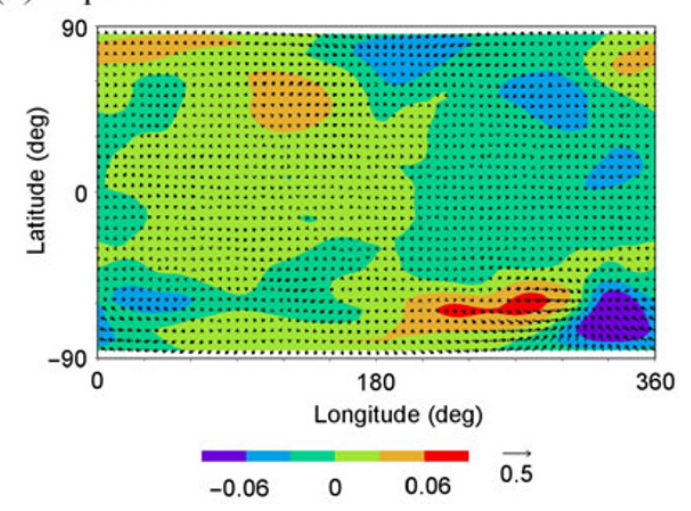

Fig. 3. Longitude-latitude distributions of eddy temperatures (K) averaged over a Venusian day in the undermost layer of $\sigma=0.995$ for (a) Exp. T and (b) Exp. NT. The arrow in the lower of the panel indicates the unit vector of eddy horizontal flow $\left(\mathrm{m} \mathrm{s}^{-1}\right)$. The superrotation flows from left to right in these panels.

Instead of orographically forced waves, the planetary-scale waves resulting from shear instability play an important role in the vertical transport of angular momentum at high latitudes (Yamamoto and Takahashi, 2006a).

The upper panels of Fig. 5 show latitude-height distributions of zonal mean flows in Exp. T and Exp. NT. The superrotation exceeding $140 \mathrm{~m} \mathrm{~s}^{-1}$ is seen near the cloud top. The wind speed is fairly fast in comparison with Exp. TY04. The faster superrotation is caused by changing the model setup. The horizontal diffusion is enhanced by 1.6 times because of reducing grid-scale noise due to topography and inertial instability, and the surface drag is changed from the Rayleigh friction formula to the bulk formula in the our study (Section 2 and Table 1). These changes unexpectedly lead to an extremely large superrotation of $>140 \mathrm{~m} \mathrm{~s}^{-1}$, although the zonal-wind acceleration/deceleration due to the horizontal diffusion is much smaller than that due to simulated waves. Around $85 \mathrm{~km}$, the zonal flow is decelerated by thermal tides and slowly propagating waves. The magnitude of the zonal flow for Exp. T is somewhat smaller than that for Exp. NT. Thus, the topographical effect on the superrotation is small in the present experiment, though the wave structures and near-surface flows (Figs. 3 and 4) are considerably different between Exp. T and Exp. NT. This finding is a significant feature of fully developed superrotation, as will be discussed in Section 4 .

The lower panels of Fig. 5 show time series of the cloud- (a) Exp. T $\left(2.7^{\circ}\right)$

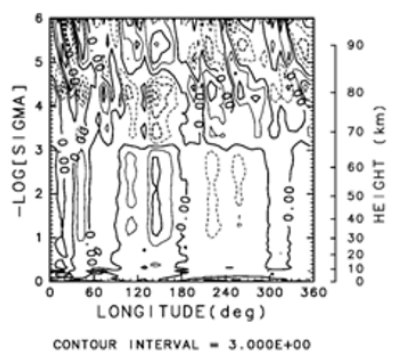

(b) Exp. T $\left(-69.2^{\circ}\right)$

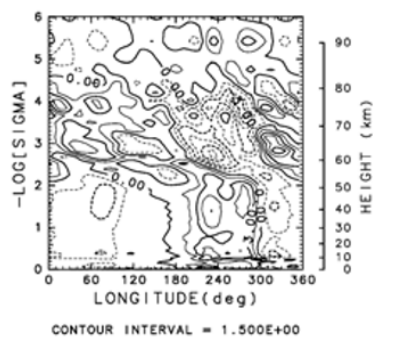

Exp. NT $\left(2.7^{\circ}\right)$

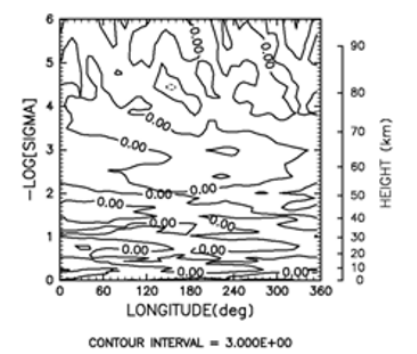

Exp. NT $\left(-69.2^{\circ}\right)$

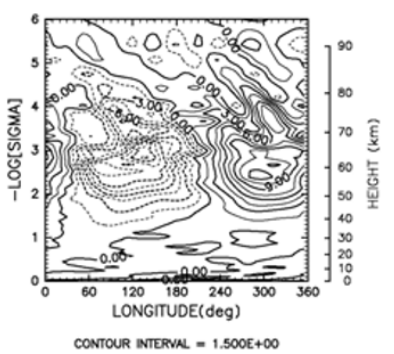

Fig. 4. Longitude-height distributions of eddy zonal flows $\left(\mathrm{m} \mathrm{s}^{-1}\right)$ averaged over a Venusian day at (a) $2.7^{\circ}$ and (b) $-69.2^{\circ}$ latitudes.

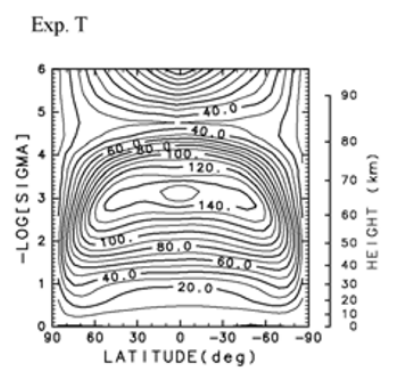

Exp. NT
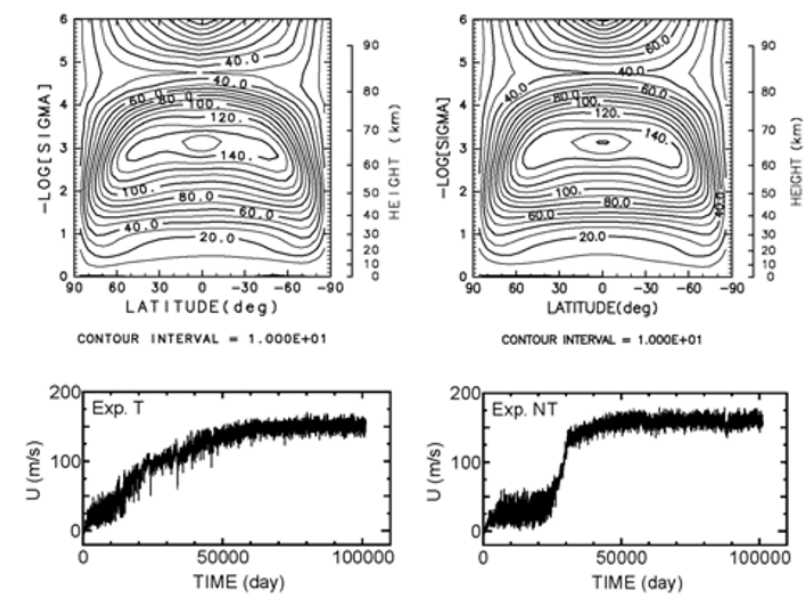

CONTOUR INIERNN $=1.000 E+01$

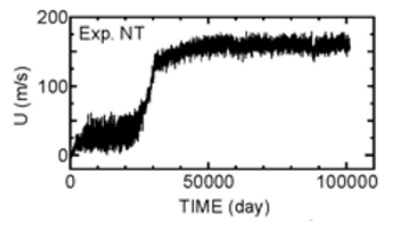

Fig. 5. Latitude-height distributions of longitudinal averaged zonal flows $\left(\mathrm{m} \mathrm{s}^{-1}\right)$ and time series of the zonal wind speed at $69 \mathrm{~km}$.

top zonal mean flows in Exp. T and Exp. NT. The cloudtop wind in Exp. NT hardly spins up until 25,000 days. After this, the wind speed rapidly increases to $150 \mathrm{~m} \mathrm{~s}^{-1}$. The rapid increase of the zonal wind occurs under the flat surface condition, when the angular momentum of dense lower atmosphere reaches the cloud top. On the other hand, the cloud-top wind in Exp. T gradually increases with time and reaches an equilibrium state around 60,000 days. In the presence of topography, the angular momentum of the dense lower atmosphere is gradually transported toward the middle atmosphere.

The meridional circulation is driven by the differential heating and the dissipation of vertically propagating waves in the same manner as our previous GCMs (Exp. YT04). The single cell of the meridional circulation is predominant in the height range from 0 to $80 \mathrm{~km}$, where the vertical flow 
is upward (downward) at the equator (the poles). Thermal tides maintain the equatorial superrotation in the middle atmosphere, together with the advection due to meridional circulation. This supports the thermal tide mechanism near the cloud top. On the other hand, horizontal shear instability plays an important role in the lower atmospheric superrotation. The equatorward eddy momentum fluxes caused by the thermal tides and shear instability (which generates 9.8day wave) deposit the angular momentum into the upward branch of the meridional circulation, in which the upward flow efficiently pumps up the angular momentum. This corresponds to the Gierasch mechanism.

\section{Summary and Discussions}

The superrotation is maintained by the Gierasch mechanism under the condition that thermal and topographic forcings are given in a Venus-like GCM. The difference between simulations with and without topography is small for the zonal mean field, though the superrotation in Exp. NT is somewhat stronger than that in Exp. T. This is largely different from Herrnstein and Dowling (2007), in which the superrotation with topography is $50 \%$ weaker than that without topography. The lowermost-level flow has a maximum velocity of $\sim 27 \mathrm{~m} \mathrm{~s}^{-1}$ in Herrnstein and Dowling's model with low vertical resolution, to which the sophisticated Planetary Boundary Layer (PBL) model using a Spalart-Allmaras scheme (Dowling et al., 2006) is applied. On the other hand, the lowermost-level flow is sufficiently decelerated by the surface drag in the thin layer between $\sigma=1$ and $0.99(\sim 100 \mathrm{~m})$ in the present study. In Herrnstein and Dowling, the orographically forced waves are likely to be generated because of large zonal surface flow; they decelerate the cloud-top superrotation because of large dissipation due to small Doppler shift velocities. Thus, significant asymmetry between northern and southern hemispheric superrotations is formed in their model. Such an asymmetry is also seen in Lee (2006). On the other hand, in the present simulation, since the orographically forced waves are weak, the topographical effect is not essential to the formation of the fully-developed superrotation. For small-scale mountains, the pressure gradient error and its related spurious winds are caused by spectral truncation and the sigma coordinate (e.g., Janjic, 1989). In our model, the small-scale (high-wavenumber) artificial disturbances are strongly dissipated by the 4th order diffusion, of which the time constant is sufficiently shorter than the radiative relaxation time near the surface. We need to evaluate the effect of the sigma coordinate on the spectral Venus model with topography.

For the stationary fields, the differences between simulations with and without topography are significant. The small-scale stationary eddies are found over the Aphrodite Terra, while the planetary-scale stationary eddies are found over Mt. Maxwell. Since the elevation of the ground surface largely changes from 0 to $\sim 10,000 \mathrm{~m}$, the thermal parameters (such as the Newtonian cooling) also largely change with elevation. Thus, weak near-surface horizontal winds flow from the mountains to the lowlands. This near-surface flow is not seen in Herrnstein and Dowling (2007), in which zonal flow and vortices are predominant. The comparison between these GCMs suggests that the near-surface flow is sensitive to model setups. In particular, the PBL parameterization (such as resolution, diffusion, and surface drag) and the pressure dependence of the thermal parameters near the surface may highly influence the pattern of the surface flow and the orographical forcing of planetary-scale stationary waves. In addition, differences with respect to the numerical methods and the coordinate system utilized in their model may be important. Thus, the sensitivities to model parameter and setup should be investigated in future studies.

Acknowledgments. Magellan topography data of Ford and Pettengill were obtained from the geosciences node of NASA's planetary data system. This study is supported by the cooperative research project of CCSR, Univ. Tokyo, and by the JSPS Grant-inAid for Young Scientists (B) (No. 17740313 and 20740273). Numerical experiments were conducted at the Information Technology Center of Univ. Tokyo and the Information Initiative Center of Hokkaido Univ. The GFD-DENNOU library is used for drawing figures.

\section{References}

Del Genio, A. D., W. Zhou, and T. P. Eichler, Equatorial superrotation in a slowly rotating GCM: Implications for Titan and Venus, Icarus, 101, $1-17,1993$.

Dowling, T. E. and coauthors, The EPIC atmospheric model with an isentropic/terrain-following hybrid vertical coordinate, Icarus, 182, 259-273, 2006

Ford, P. G. and G. H. Pettengill, Venus topography and kilometer-scale slopes, J. Geophys. Res., 97, 13103-13114, 1992.

Gierasch, P. J., Meridional circulation and the maintenance of the Venus atmospheric rotation, J. Atmos. Sci., 32, 1038-1044, 1975.

Herrnstein, A. and T. E. Dowling, Effects of topography on the spinup of a Venus atmospheric model, J. Geophys. Res., 112, E04S08, doi:10.1029/2006JE002804, 2007.

Hollingsworth, J. L. and coauthors, A simple-physics global circulation model for Venus: Sensitivity assessments of atmospheric superrotation, Geophys. Res. Lett., 34, L05202, doi:10.1029/2006GL028567, 2007.

Iga, S. and Y. Matsuda, Shear instability in a shallow water model with implication for the Venus atmosphere, J. Atmos. Sci., 62, 2514-2527, 2005.

Janjic, Z. I., On the pressure gradient force error in $\sigma$-coordinate spectral models, Mon. Wea. Rev., 117, 2285-2292, 1989.

Lee, C., Modelling of the atmosphere of Venus, Ph.D. thesis, Oxford University, pp. 216, 2006.

Lee, C., S. R. Lewis, and P. L. Read, A numerical model of the atmosphere of Venus, Adv. Space Res., 36, 2142-2145, 2005.

Newman, M. and C. B. Leovy, Maintenance of strong rotational winds in Venus' middle atmosphere by thermal tides, Science, 257, 647-650, 1992.

Numaguti, A. and coauthors, Study on the climate system and mass transport by a climate model, CGER's Supercomputer monograph report, 3 , pp. 91, 1997.

Rossow, W. B. and G. P. Williams, Large-scale motion in the Venus stratosphere, J. Atmos. Sci., 36, 377-389, 1979.

Yamamoto, M. and M. Takahashi, The fully developed superrotation simulated by a general circulation model of a Venus-like atmosphere, $J$. Atmos. Sci., 60, 561-574, 2003.

Yamamoto, M. and M. Takahashi, Dynamics of Venus' superrotation: the eddy momentum transport processes newly found in a GCM, Geophys. Res. Lett., 31, L09701, doi:10.1029/2004GL019518, 2004.

Yamamoto, M. and M. Takahashi, Superrotation maintained by meridional circulation and waves in a Venus-like AGCM, J. Atmos. Sci., 63, 32963314, 2006a.

Yamamoto, M. and M. Takahashi, Stationary and slowly propagating waves in a Venus-like AGCM: Roles of topography in Venus' atmospheric dynamics, Theor. Appl. Mech. Jpn., 55, 201-207, 2006 b.

M. Yamamoto (e-mail: yamakatu@riam.kyushu-u.ac.jp) and M Takahashi 